Joel M. Gichumbi, Bernard Omondi* and Holger B. Friedrich

\title{
Crystal structure of $\eta^{6}$-p-cymene-iodido-( $N$-isopropyl-1-(pyridin-2-yl) methanimine- $\left.{ }^{2} N, N^{\prime}\right)$ ruthenium(II) hexafluorophosphate(V), $\mathrm{C}_{19} \mathrm{H}_{26} \mathrm{IN}_{2} \mathrm{~F}_{6} \mathrm{Ru}$
}

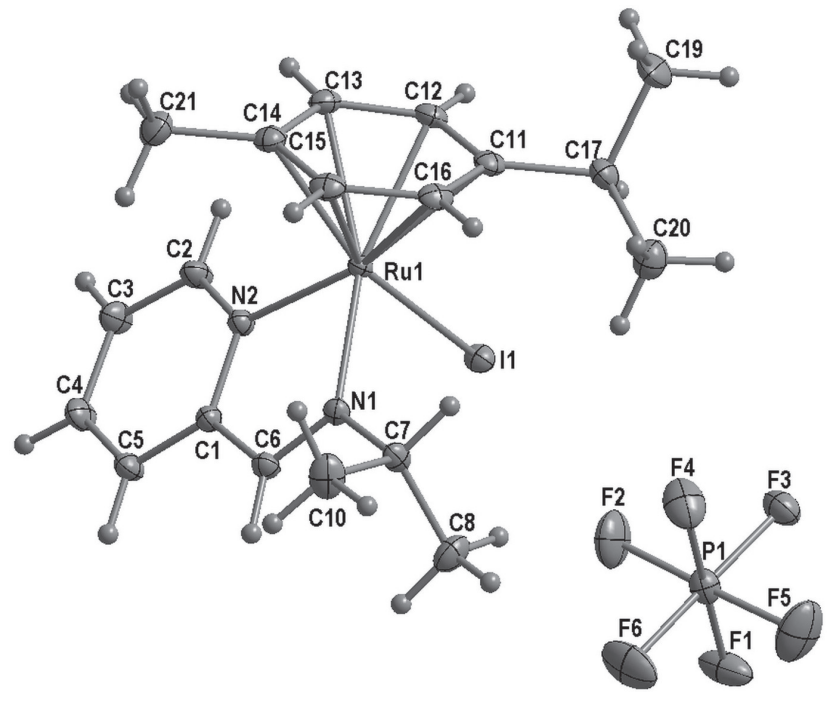

https://doi.org/10.1515/ncrs-2019-0786

Received October 22, 2019; accepted November 11, 2019; available online December 7, 2019

\begin{abstract}
$\mathrm{C}_{19} \mathrm{H}_{26} \mathrm{IN}_{2} \mathrm{~F}_{6} \mathrm{Ru}, \quad$ triclinic, $P \overline{1} \quad$ (no. 2), $a=9.240$ (2) $\AA$, $b=10.019$ (3) $\AA, c=12.689$ (3) $\AA, \alpha=78.921^{\circ}, \beta=81.596(10)^{\circ}$, $\gamma=83.654^{\circ}, \quad V=1136.30(5) \AA^{3}, \quad Z=2, \quad R_{\mathrm{gt}}(F)=0.0174$, $w R_{\text {ref }}\left(F^{2}\right)=0.0440, T=173 \mathrm{~K}$.
\end{abstract}

\section{CCDC no.: 1964837}

The asymmetric unit of the salt-type title is shown in the figure. Table 1 contains crystallographic data and Table 2 contains the list of the atoms including atomic coordinates and displacement parameters.

\footnotetext{
*Corresponding author: Bernard Omondi, University of KwaZulu-Natal, School of Chemistry and Physics, P.O. Box X01, Pietermaritzburg Campus, Scottsville, Pietermaritzburg 3209, South Africa, e-mail: owaga@ukzn.ac.za. https://orcid.org/00000002-3003-6712

Joel M. Gichumbi: Chuka University, Department of Physical Sciences, P.O. Box 109-60400, Chuka, Kenya

Holger B. Friedrich: University of KwaZulu-Natal, School of Chemistry and Physics, PO Box X54001, Westville Campus, Westville 4000, Durban, South Africa
}

Table 1: Data collection and handling.

\begin{tabular}{ll}
\hline Crystal: & Orange block \\
Size: & $0.20 \times 0.17 \times 0.14 \mathrm{~mm}$ \\
Wavelength: & Mo $K \alpha$ radiation $(0.71073 \AA)$ \\
$\mu:$ & $2.18 \mathrm{~mm}^{-1}$ \\
Diffractometer, scan mode: & Bruker Smart Apex-II, $\varphi$ and $\omega$ \\
$\theta_{\max }$, completeness: & $28.3^{\circ},>99 \%$ \\
$N(h k l)_{\text {measured }}, N(h k l)_{\text {unique }}, R_{\text {int }}:$ & $17004,5626,0.015$ \\
Criterion for $I_{\text {obs }}, N\left(h k l_{\mathrm{gt}}:\right.$ & $I_{\text {obs }}>2 \sigma\left(I_{\text {obs }}\right), 5236$ \\
$N(\text { param })_{\text {refined }}:$ & 271 \\
Programs: & Bruker [1], SHELX [2], \\
& WinGX/ORTEP [3] \\
\hline
\end{tabular}

\section{Source of material}

To a suspension of $\left[\left(\eta^{6}-p \text {-cymene }\right) \mathrm{Ru}(\mu-\mathrm{I}) \mathrm{I}\right]_{2}(0.2 \mathrm{mmol})$ in methanol $(20 \mathrm{~mL})$ was added the ligand $(0.42 \mathrm{mmol})$. The mixture was stirred at room temperature for $3 \mathrm{~h}$ followed by reduction of the solvent volume in vacuo to about $(10 \mathrm{~mL})$ before adding $\mathrm{NH}_{4} \mathrm{PF}_{6}(0.42 \mathrm{mmol})$. The mixture was then cooled in an ice bath while stirring for $2 \mathrm{~h}$ leading to a precipitate, which was collected by filtration. The filtrate was washed with diethyl ether and dried in vacuo. Crystals were grown by the liquid diffusion method by layering a solution of the compound in acetone with hexane and left undisturbed for 2 days. Orange crystals, yield $=80 \%$, m.p. $150{ }^{\circ} \mathrm{C}$ (decomp.).

${ }^{1} \mathbf{H}$ NMR (400 MHZ, DMSO-d $\left.\mathrm{d}_{6}\right) . \delta[\mathrm{ppm}] 9.51(\mathrm{~d}$, $\left.J_{\mathrm{HH}}=5.4 \mathrm{~Hz}, 1 \mathrm{H}, \mathrm{Py}\right) ; 8.80(\mathrm{~s}, 1 \mathrm{H}, \mathrm{CH}=\mathrm{N}) ; 8.23-8.21(\mathrm{M}$, $1 \mathrm{H}, \mathrm{Py}) ; 8.15\left(\mathrm{~d}, J_{\mathrm{HH}}=7.2 \mathrm{~Hz}, 1 \mathrm{H}, \mathrm{Py}\right) ; 7.80(\mathrm{~s}, 1 \mathrm{H}, \mathrm{Py})$; $6.26\left(\mathrm{~d}, J_{\mathrm{HH}}=6.26 \mathrm{~Hz}, 1 \mathrm{H},(\mathrm{p}-\mathrm{cyAr})\right) ; 6.18\left(\mathrm{~d}, J_{\mathrm{HH}}=6.10 \mathrm{~Hz}\right.$, $1 \mathrm{H}\left(\mathrm{p}\right.$-cyAr)); $5.94\left(\mathrm{~d}, J_{\mathrm{HH}}=6.10 \mathrm{~Hz}, 1 \mathrm{H},(\mathrm{p}-\mathrm{cyAr})\right), 5.89(\mathrm{~d}$, $\left.J_{\mathrm{HH}}=6.30 \mathrm{~Hz}, 1 \mathrm{H},(\mathrm{p}-\mathrm{cyAr})\right), 4.88(\mathrm{sep}, 1 \mathrm{H}, \mathrm{CH}) 2.63(\mathrm{~m}, 1 \mathrm{H}, \mathrm{CH}$ (Me)2); 2.16 (s, 3H, (Me)); 1.59 (d, $J_{\mathrm{HH}}=6.80 \mathrm{~Hz}, 3 \mathrm{H},(\mathrm{Me}) ; 1.41$ $\left(\mathrm{d}, J_{\mathrm{HH}}=6.64 \mathrm{~Hz}, 3 \mathrm{H},(\mathrm{Me})\left(0.95\left(\mathrm{~d}, J_{\mathrm{HH}}=6.88 \mathrm{~Hz}, 3 \mathrm{H},(\mathrm{Me})\right.\right.\right.$. ${ }^{13}$ C NMR (400 MHZ, DMSO-d 6 ). $\delta$ [ppm] $165.3(\mathrm{CH}=\mathrm{N}), 155.7$ (Рy), 155.5 (Рy); 139.8 (Рy); 128.8 (Рy); 128.2 (Py); 104.2 (Ar); 103.4 (Ar); 87.6 (Ar); 84.5 (Ar) ; $65.5(\mathrm{CH})$; $30.5(\mathrm{Me}) ; 23.8(\mathrm{Me})$; $22.3(\mathrm{Me}) ; 22.1(\mathrm{Me}) ; 21.30(\mathrm{Me}) ; 18.4(\mathrm{Me})$. IR (solid state): $\gamma$ $(\mathrm{C}=\mathrm{N}) 1622.1 \mathrm{~cm}^{-1}$. MS (ESI, M/Z): $520.98\left[\mathrm{C}_{19} \mathrm{H}_{26} \mathrm{IN}_{2} \mathrm{Ru}\right]^{+}$.

\section{Experimental details}

Crystal evaluation and data collection were done on a Bruker Smart APEX2 diffractometer equipped with an Oxford 
Table 2: Fractional atomic coordinates and isotropic or equivalent isotropic displacement parameters $\left(\AA^{2}\right)$.

\begin{tabular}{|c|c|c|c|c|}
\hline Atom & $x$ & $y$ & $z$ & $\boldsymbol{U}_{\text {iso }}{ }^{*} / \boldsymbol{U}_{\mathrm{eq}}$ \\
\hline $\mathrm{C} 1$ & $0.34230(19)$ & $0.25218(17)$ & $0.70043(14)$ & $0.0138(3)$ \\
\hline C2 & $0.3932(2)$ & $0.02407(18)$ & $0.77376(15)$ & $0.0182(3)$ \\
\hline $\mathrm{H} 2$ & 0.4523 & -0.0454 & 0.8139 & $0.022^{\star}$ \\
\hline C3 & $0.2694(2)$ & $-0.01053(19)$ & $0.73865(16)$ & $0.0202(4)$ \\
\hline H3 & 0.2457 & -0.1027 & 0.7535 & $0.024^{\star}$ \\
\hline $\mathrm{C} 4$ & $0.1804(2)$ & $0.0900(2)$ & $0.68173(16)$ & $0.0201(4)$ \\
\hline H4 & 0.0967 & 0.0675 & 0.6555 & $0.024^{\star}$ \\
\hline $\mathrm{C} 5$ & $0.2159(2)$ & $0.22430(19)$ & $0.66384(15)$ & $0.0175(3)$ \\
\hline H5 & 0.1551 & 0.2958 & 0.6273 & $0.021^{\star}$ \\
\hline C6 & $0.39037(19)$ & $0.38808(17)$ & $0.68729(14)$ & $0.0146(3)$ \\
\hline H6 & 0.3355 & 0.4662 & 0.6532 & $0.017^{*}$ \\
\hline $\mathrm{C} 7$ & $0.5638(2)$ & $0.53641(17)$ & $0.71118(14)$ & $0.0156(3)$ \\
\hline H7 & 0.6556 & 0.5258 & 0.7462 & 0.019 * \\
\hline $\mathrm{C} 8$ & $0.4519(2)$ & $0.6296(2)$ & $0.76871(18)$ & $0.0253(4)$ \\
\hline $\mathrm{H} 8 \mathrm{~A}$ & 0.4888 & 0.7196 & 0.7595 & $0.038^{\star}$ \\
\hline $\mathrm{H} 8 \mathrm{~B}$ & 0.3593 & 0.6387 & 0.7378 & $0.038^{\star}$ \\
\hline $\mathrm{H} 8 \mathrm{C}$ & 0.4352 & 0.5905 & 0.8460 & $0.038^{\star}$ \\
\hline C10 & $0.6023(2)$ & $0.5951(2)$ & $0.59182(16)$ & $0.0253(4)$ \\
\hline $\mathrm{H} 10 \mathrm{~A}$ & 0.6371 & 0.6857 & 0.5844 & $0.038^{\star}$ \\
\hline $\mathrm{H} 10 \mathrm{~B}$ & 0.6796 & 0.5348 & 0.5593 & $0.038^{\star}$ \\
\hline $\mathrm{H} 10 \mathrm{C}$ & 0.5149 & 0.6025 & 0.5549 & $0.038^{\star}$ \\
\hline C11 & $0.81503(18)$ & $0.21185(18)$ & $0.88233(15)$ & $0.0158(3)$ \\
\hline C12 & $0.76943(19)$ & $0.07682(18)$ & $0.89261(15)$ & $0.0160(3)$ \\
\hline H12 & 0.7500 & 0.0231 & 0.9626 & $0.019^{\star}$ \\
\hline C13 & $0.75308(19)$ & $0.02281(18)$ & $0.80155(15)$ & $0.0167(3)$ \\
\hline $\mathrm{H} 13$ & 0.7274 & -0.0683 & 0.8107 & 0.020 * \\
\hline C14 & $0.77435(19)$ & $0.10265(19)$ & $0.69490(15)$ & $0.0176(3)$ \\
\hline C15 & $0.81764(19)$ & $0.23640(19)$ & 0.68479 & $0.0178(3)$ \\
\hline H15 & 0.8322 & 0.2921 & 0.6 & 0.021 * \\
\hline C16 & $0.83962(18)$ & $0.28840(19)$ & $0.77760(15)$ & $0.0167(3)$ \\
\hline H16 & 0.8718 & 0.3773 & 0.7683 & 0.020 * \\
\hline C17 & $0.8355(2)$ & $0.26487(19)$ & $0.98273(15)$ & $0.0191(4)$ \\
\hline $\mathrm{H} 17$ & 0.7565 & 0.2309 & 1.0413 & $0.023^{*}$ \\
\hline C19 & $0.9831(3)$ & $0.2031(3)$ & $1.0193(2)$ & $0.0391(6)$ \\
\hline $\mathrm{H} 19 \mathrm{~A}$ & 0.9973 & 0.2369 & 1.0843 & $0.059^{\star}$ \\
\hline H19 & 0.9839 & & & $0.059^{*}$ \\
\hline $\mathrm{H} 19 \mathrm{C}$ & 1.0626 & 0.2296 & 0.9614 & $0.059^{\star}$ \\
\hline $\mathrm{C} 20$ & $0.8230(2)$ & $0.4203(2)$ & $0.96849(17)$ & $0.0264(4)$ \\
\hline $\mathrm{H} 20 \mathrm{~A}$ & 0.8374 & 0.4472 & 1.0363 & 0.040 * \\
\hline $\mathrm{H} 20 \mathrm{~B}$ & 0.8982 & 0.4568 & 0.9106 & $0.040^{\star}$ \\
\hline $\mathrm{H} 20 \mathrm{C}$ & 0.7255 & 0.4566 & 0.9495 & $0.040^{\star}$ \\
\hline $\mathrm{C} 21$ & $0.7496(2)$ & $0.0468(2)$ & $0.59780(16)$ & $0.0243(4)$ \\
\hline $\mathrm{H} 21 \mathrm{~A}$ & 0.7204 & -0.0466 & 0.6210 & $0.036^{\star}$ \\
\hline $\mathrm{H} 21 \mathrm{~B}$ & 0.6717 & 0.1042 & 0.5619 & $0.036^{\star}$ \\
\hline $\mathrm{H} 21 \mathrm{C}$ & 0.8405 & 0.0462 & 0.5472 & $0.036^{\star}$ \\
\hline N1 & $0.51049(16)$ & $0.39793(14)$ & $0.72382(11)$ & $0.0129(3)$ \\
\hline N2 & $0.43209(16)$ & $0.15269(15)$ & $0.75287(12)$ & $0.0135(3)$ \\
\hline P1 & $-0.01712(5)$ & $0.32867(5)$ & $1.35346(4)$ & $0.01770(9)$ \\
\hline Ru1 & $0.61542(2)$ & $0.21849(2)$ & $0.79809(2)$ & $0.01065(4)$ \\
\hline 11 & $0.43390(2)$ & $0.25309(2)$ & $0.97949(2)$ & $0.01582(4)$ \\
\hline F1 & $-0.17631(14)$ & $0.34985(15)$ & $1.42094(10)$ & $0.0367(3)$ \\
\hline F2 & $-0.06014(17)$ & $0.19179(15)$ & $1.32491(12)$ & $0.0408(3)$ \\
\hline F3 & $0.04199(13)$ & $0.24255(13)$ & $1.46124(10)$ & $0.0267(3)$ \\
\hline F4 & $0.14242(14)$ & $0.30624(16)$ & $1.28689(11)$ & $0.0358(3)$ \\
\hline F5 & $0.0292(2)$ & $0.46406(14)$ & $1.38355(13)$ & $0.0490(4)$ \\
\hline F6 & $-0.07647(17)$ & $0.41510(17)$ & $1.24738(11)$ & $0.0451(4)$ \\
\hline
\end{tabular}

Cryostream low temperature apparatus operating at 100(1) K. The structure was solved by direct method using the SHELXS [2] program and refined with SHELXL [2]. All hydrogen atoms were placed in idealized positions and refined in riding models with $U_{\text {iso }}$ assigned the values of 1.2 times those of their parent atoms and the distances of $\mathrm{C}-\mathrm{H}$ were constrained to $0.93 \AA$ for all the aromatic H atoms, $0.960 \AA$ A for methyl hydrogens and $0.98 \AA$ for methine hydrogen. The visual crystal structure information was performed using ORTEP-3 [3].

\section{Comment}

Ruthenium half-sandwich complexes have found many applications as metallodrugs and as catalysts in various reactions [4, 5]. Biologically, ruthenium complexes have shown potential as anticancer agents $[6,7]$ and as antimicrobial agents [6]. In catalysis, ruthenium half-sandwich complexes have found applications in, oxidative transformations of alkanes [4], cleavage of double bonds [8], oxidation of alcohols [9] and in transfer hydrogen of ketones [10].

The title compound is part of our continuing studies of half-sandwich ruthenium(II) complexes [6-11]. The asymmetric unit contains one cationic ruthenium complex featuring a "pseudo-octahedral three-legged piano stool" structure and one counter anion (see the figure). In this arrangement, the ruthenium centre is coordinated to the $N, N^{\prime}$-bidentate ligand, through the $\mathrm{N}$ atom of the pyridine moeity and the imine $\mathrm{N}$ atom, and one iodido ligand to form the base of the stool while the $p$-cymene ring acts as the apex of the stool [6-10]. In the complex, the $\mathrm{Ru}-\mathrm{N}$ bond distances are 2.0714(14) and 2.0761(14) $\AA$. This value is comparable to those reported for other arene ruthenium complexes with $N, N^{\prime}$ donor ligands [4-10]. The $\mathrm{N}-\mathrm{Ru}-\mathrm{N}$ bond angle is 76.99(6) while those of $\mathrm{N}-\mathrm{Ru}-\mathrm{I}$ are $84.44(4)$ and $83.95(4)^{\circ}$. These values are close to those reported for related compounds $[6,12]$. All $\mathrm{C}-\mathrm{C}, \mathrm{C}-\mathrm{N}$ and $\mathrm{P}-\mathrm{F}$ bond length and angles are in the expected range [6-13].

Acknowledgements: We wish to extend our sincere thanks to the NRF, THRIP (Grant no. Tp 1208035643) and UKZN (URF) for financial support. Gichumbi, M. Joel thanks Prof. E. N. Njoka for his support.

\section{References}

1. Bruker. APEXII. Bruker AXS Inc, Madison, WI, USA (2009).

2. Sheldrick, G. M.: A short history of SHELX. Acta Crystallogr. A64 (2008) 112-122.

3. Farrugia, L. J.: WinGX and ORTEP for Windows. Appl. Crystallogr. 45 (2012) 849-854.

4. Gichumbi, J. M.; Friedrich, H. B.: Half-sandwich complexes of platinum complexes of platinum group metals (Ir, Rh, Ru and 
Os) and some recent biological and catalytic applications. J. Organomet. Chem. 866 (2018) 123-143.

5. Kumar, P.; Gupta, R.: Half-sandwich arene ruthenium complexes: synthetic strategies and relevance in catalysis. Chem. Soc. Rev. 43 (2014) 707-733.

6. Gichumbi, J. M.; Omondi, B.; Lazarus, G.; Singh, M.; Shaikh, N.; Chenia, H. Y.; Friedrich, H. B.: Influence of halogen substitution in the ligand sphere on the antitumor and antibacterial activity of half-sandwich ruthenium(II) complexes [RuX $\left(\eta^{6}-\right.$ arene) $\left.\left(\mathrm{C}_{5} \mathrm{H}_{4} \mathrm{~N}-2-\mathrm{CH}=\mathrm{N}-\mathrm{Ar}\right)\right]^{+}$. Z. Anorg. Allg. Chem. 643 (2017) 699-711.

7. Thangaval, S.; Paulpandi, M.; Friedrich, H. B.; Murugan, K.; Kalva, S.; Skelton, A. A.: Synthesis, characterization, antiproliferative and molecular docking study of new half sandwich Ir(III), Rh(III) and Ru(II) complexes, J. Inorg. Biochem. 159 (2016) 50-61.

8. Gichumbi, J. M.; Friedrich, H. B.; Omondi, B.: Synthesis and characterization of piano-stool ruthenium complexes with $N, N^{\prime}$ - pyridine imine bidentate ligands and their application in styrene oxidation. J. Organomet. Chem. 808 (2016) 87-96.

9. Gichumbi, J. M.; Friedrich, H. B.; Omondi, B.: Synthesis and characterization of some new half-sandwich ruthenium(II) complexes with bidentate $N, N^{\prime}$-ligands and their application in alcohol oxidation. Inorg. Chim. Acta. 456 (2017) 55-63.

10. Gichumbi, J. M.; Friedrich, H. B.; Omondi, B.: Application of arene ruthenium(II) complexes with pyridine-2-carboxaldimine ligands in the transfer hydrogenation of ketones. J. Mol. Catal. A: Chem. 416 (2016) 29-38.

11. Nyawade, E. A.; Friedrich, H. B.; Omondi, B.; Mpungose, P.: Synthesis and characterization of new ( $\eta^{5}$-cyclopentadienyl) dicarbonylruthenium(II) amine complexes: their application as homogeneous catalysts in styrene oxidation. Organometallics 34 (2015) 4922-4931.

12. Betanzos-Lara, S.; Novakova, O.; Deeth, R. J.; Pizarro, A. M.; Clarkson, G. J.; Liskova, B.; Brabec, V.; Sadler, P. J.; Habtemariam, A.: Bipyrimidine ruthenium(II) arene complexes: structure, reactivity and cytotoxicity. J. Biol. Inorg. Chem. 17 (2012) 1033-1051.

13. Gichumbi, J. M.; Omondi, B.; Friedrich, H. B.: Crystal structure of chlorido-( $\eta^{6}-1$-isopropyl-4-methyl benzene)-(1-(pyridin2-yl)- $N$-( $p$-tolyl)methanimine- $\left.{ }^{2} N, N^{\prime}\right)$ ruthenium(II) hexafluorophosphate(V), $\mathrm{C}_{23} \mathrm{H}_{26} \mathrm{ClF}_{6} \mathrm{~N}_{2}$ PRu. Z. Kristallogr. NCS 232 (2017) 285-287. 